Recepción: 08 / 01 / 2018

Aceptación: 19 / 02 / 2018

Publicación: 21 / 04 / 2018

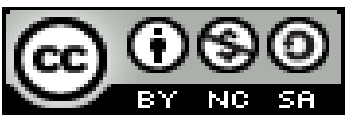

Ciencias económicas y empresariales

Artículo de Revisión

\title{
La gestión de los recursos y capacidades empresariales, y su relación con la calidad del servicio al cliente en el mercado farmacéutico de la ciudad de Loja
}

The management of resources and business capabilities, and its relationship with the quality of customer service in the pharmaceutical market of the city of Loja

\section{A gestão de recursos e capacidades de negócios, e sua relação com a qualidade do atendimento ao cliente no mercado farmacêutico da cidade de Loja}

\author{
Ney Alfredo Gallardo ${ }^{\mathrm{I}}$ \\ ney.gallardo@unl.edu.ec \\ Manuel E. Pasaca-Mora II \\ manuel.pasaca@unl.edu.ec \\ Rocío del C. Toral-Tinitana III \\ rocio.toral@unl.edu.ec \\ Carmen Cevallos-Cueva IV \\ carmen.cevallos@unl.ed.ec
}

Correspondencia: ney.gallardo@unl.edu.ec

\footnotetext{
I Magíster en Docencia Universitaria, Doctorado en Ciencias Administrativas, PhD. (Tesista), Licenciado en Administración de Empresas, Ingeniero Comercial, Docente Titular Principal de la Universidad Nacional de Loja, Loja, Ecuador.

${ }^{\text {II }}$ Magíster en Docencia Universitaria en el Sistema Modular, Licenciado en Administración de Empresas, Ingeniero Comercial, Docente Titular Principal de la Universidad Nacional de Loja, Loja, Ecuador.

III Magíster en Docencia Universitaria e Investigación Educativa, Magister en Administración de Empresas, Licenciada en Administración de Empresas, Ingeniera Comercial, Docente Titular Principal de la Universidad Nacional de Loja, Loja, Ecuador.

IV Magíster en Docencia Universitaria e Investigación Educativa, Magister en Administración de Empresas, Diplomado Superior en Planificación de Desarrollo Local, Diploma Superior en Gestión del Talento Humano, Licenciada en Administración de Empresas, Ingeniera Comercial, Docente Titular Principal de la Universidad Nacional de Loja, Loja, Ecuador.
} 


\section{Resumen}

El estudio de la variable Gestión de los recursos y capacidades, midió las percepciones del personal directivo y del personal del servicio de atención al cliente, sobre la gestión de los recursos y capacidades directivas, la gestión de los recursos y capacidades de marketing, la gestión de los recursos y capacidades financieras, la gestión de los recursos y capacidades del potencial humano y la gestión de recursos y capacidades de operación de las farmacias de la ciudad de Loja, y la variable calidad de los servicios, se midió las percepciones del personal administrativo y del personal del servicio de atención al cliente, sobre tangibilidad, fiabilidad, capacidad de respuesta, seguridad y empatía. Los cuestionarios se aplicaron a una población de 204 personas, de los cuales 68 son directivos de la empresa y 136 son personal del servicio de atención al cliente. El estudio determina que para los clientes la gestión de recursos y capacidades, logra una valoración favorable, de acuerdo al promedio cercano a los 3 puntos. Al tratarse de los aspectos relacionados a la calidad del servicio, los promedios cercanos a los 3 puntos dejan en evidencia generalmente que los clientes tienen una percepción favorable de los elementos tangibles de la fiabilidad, capacidad de respuesta y empatía del servicio. Al referirse a la relación entre la gestión de los recursos y capacidades directivas y la calidad del servicio, se observa que los clientes que califican mejor la gestión de los recursos y capacidades directivas, son los que mejor califican a la calidad del servicio, esto es el $51.5 \%$ y $57.1 \%$ respectivamente. $\mathrm{Al}$ analizar de manera global la gestión administrativa, los resultados muestran, que los clientes califican a dicho tipo de gestión como buena o muy buena, de acuerdo a la opinión del 59.2\% y $100 \%$, respectivamente. Al final se llega a determinar que la gestión administrativa incide significativa (Sig. $<0.059$ ) en la satisfacción del cliente. La correlación positiva por su parte indica que en la medida que mejora la calificación de la gestión administrativa, mejorará la satisfacción del cliente. Las deducciones citadas nos permiten aceptar la hipótesis, en que, la gestión de los recursos y capacidades se relacionan significativamente con la calidad de los servicios de atención al cliente en el mercado farmacéutico de la ciudad de Loja.

Palabras clave: gestión; recursos; capacidades; calidad del servicio; atención al cliente; mercado farmacéutico. 


\section{Abstract}

The study of the variable Management of the resources and capacities, measured the perceptions of the managerial staff and of the personnel of the service of the customer service, on the management of the resources and managerial capacities, the management of the resources and capacities of marketing, the management of the resources and financial capacities, the management of the resources and capacities of the human potential and the management of resources and operation capacities of the pharmacies of the city of Loja, and the variable quality of the services, the perceptions of the administrative personnel were measured and the customer service staff, about tangibility, reliability, responsiveness, security and empathy. The questionnaires were applied to a population of 204 people, of whom 68 are managers of the company and 136 are customer service personnel. The study determines that for the clients the management of resources and capacities, achieves a favorable valuation, according to the average close to 3 points. When dealing with the aspects related to the quality of service, the averages close to the 3 points usually show that clients have a favorable perception of the tangible elements of reliability, response capacity and service empathy. When referring to the relationship between the management of resources and managerial skills and the quality of the service, it is observed that the clients that best qualify the management of resources and managerial capacities, are those that best qualify the quality of the service, this is $51.5 \%$ and $57.1 \%$ respectively. When analyzing overall administrative management, the results show that customers rate this type of management as good or very good, according to the opinion of 59.2\% and 100\%, respectively. In the end, it is determined that administrative management has a significant impact (Sig. <0.059) on customer satisfaction. The positive correlation on the other hand indicates that to the extent that it improves the qualification of the administrative management, it will improve the satisfaction of the client. The aforementioned deductions allow us to accept the hypothesis, in which the management of resources and capabilities are significantly related to the quality of customer services in the pharmaceutical market of the city of Loja.

Keywords: Management; resources; capabilities; quality of service; Customer Support; pharmaceutical market. 


\section{Resumo}

O estudo dos recursos de gestão variáveis e capacidades, percepções de gestão e pessoal de serviço ao cliente medido na gestão dos recursos e competências de gestão, gestão de recursos e capacidades de marketing, gestão de recursos e capacidades financeiras, gestão de recursos e capacidades do potencial humano e gestão de recursos e capacidades operacionais de farmácias na cidade de Loja, e a qualidade variável dos serviços, as percepções do pessoal administrativo foi medida e a equipe de atendimento ao cliente, sobre tangibilidade, confiabilidade, capacidade de resposta, segurança e empatia. Os questionários foram aplicados a uma população de 204 pessoas, das quais 68 são gerentes da empresa e 136 são funcionários de atendimento ao cliente. $\mathrm{O}$ estudo determina que para os clientes a gestão de recursos e capacidades, alcança uma valorização favorável, segundo a média próxima a 3 pontos. Sendo aspectos relacionados à qualidade de serviço, perto dos 3 pontos em evidência média licença que os clientes geralmente têm uma percepção favorável dos elementos tangíveis de confiabilidade, capacidade de resposta e empatia de serviço. Referindo-se à relação entre a gestão de recursos e competências de gestão e qualidade do serviço, mostra que os clientes melhor qualificados recursos de gestão e técnicas de gestão, são mais qualificados para a qualidade do serviço, este é 51,5\% e 57,1\% respectivamente. Ao analisar a gestão administrativa global, os resultados mostram que os clientes classificam este tipo de gestão como bom ou muito bom, de acordo com a opinião de 59,2\% e 100\%, respectivamente. No final, é determinado que o gerenciamento administrativo tem um impacto significativo (Sig. <0,059) na satisfação do cliente. A correlação positiva, por outro lado, indica que, na medida em que melhora a qualificação da gestão administrativa, melhorará a satisfação do cliente. Deduções citado nos permite aceitar a hipótese de que a gestão dos recursos e capacidades estão significativamente relacionadas com a qualidade dos serviços ao cliente no mercado farmacêutico na cidade de Loja.

Palavras chave: Gestão; recursos; capacidades; qualidade de serviço; atendimento ao cliente; mercado farmacêutico.

\section{Introducción}

En la ciudad de Loja, la gestión empresarial de las farmacias tiene varios condicionantes que deben consolidarse. Por un lado, las farmacias desempeñan un papel fundamental en el canal de distribución del sector farmacéutico, dado que son el principal eslabón entre el mayorista y los 
consumidores o usuarios finales. El número de puntos de venta en la ciudad de Loja es de 68 farmacias y su volumen de negocio es considerable. Su gestión está condicionada por las actuaciones de la administración pública, pues parte de los medicamentos son distribuidos por el sistema nacional de salud y obligan que se los expenda en las farmacias (medicina genérica), esto genera preocupación en el cliente por el bajo efecto que producen.

El presente trabajo, permite expresar la gestión administrativa en el mercado farmacéutico de la ciudad de Loja, para ello se muestran antecedentes en el que se incluye una revisión de trabajos relacionados a la presente investigación, entre los que se destaca por el aporte que brindan; y se complementa con el marco teórico que permite el sustento fundamental de la variable gestión de los recursos y capacidades empresariales; se detalla la metodología aplicada en la investigación; se incluye los resultados del análisis de las variables y la discusión de los mismos en relación a otras investigaciones efectuadas, y, la teoría expuesta como referente.

Como relativos para la elaboración del trabajo se apeló a trabajos afines de ciertos autores, entre ellos Pérez (2002) "La satisfacción al cliente como ventaja competitiva en farmacias de autoservicio de Venezuela", Pérez (2008) "La satisfacción al cliente en farmacias de autoservicio de San Juan de los Morros, Estado Guárico", Morales (2010) "Estudio para determinar la calidad de la atención que brinda la farmacia de emergencia del Hospital María Auxiliadora de Lima a los usuarios externos", Castellanos, Susie y González, Pamela (2010) "La calidad de los servicios en farmacias con enfoque tradicional y las de autoservicio en el municipio de Maracaibo", Soto (2011) "la gestión administrativa en centros médicos auspiciados por organismos internacionales, caso club rotario", Taipe (2012) "Sistema de Gestión Estratégica para las Cuentas por Cobrar en la Farmacia Cruz Azul Nº1, período del 1de enero al 30 de junio del 2009”, González y Bermúdez (2016) "la calidad de los servicios farmacéuticos comunitarios del área de salud Julián Grimau, en el municipio Santiago de Cuba, en el período comprendido entre enero y febrero del 2010"; por otro lado se incluye las categorías teóricas sobre la variable estudiada.

Entre las limitantes que se afrontó en el trabajo, es el no poder comparar los resultados del presente trabajo con otros ya realizados por cuanto, el procedimiento que dan los autores a sus trabajos, no tienen relación con los objetivos planteados en el presente. 
El marco teórico en relación a la Gestión se acudió a las propuestas de ciertos autores como son: Ivnacevich y Lorenzi (2006), Laborda, Leopoldo; De Zuani, Elio (2009), Campos, Segundo y Loza, Pedro (2011), Medina (2012); Al referirse a la Calidad, tenemos a los autores; Ivnacevich y Lorenzi (2006), Fragas (2012), Yamaguchi (1989), Feigenbaum (1971-1994), Hansen (1996) citado por López (2005).

Este trabajo, procura establecerse en una prueba de lo que actualmente constituye la relación entre la gestión administrativa y la calidad de los servicios de atención al cliente en el mercado farmacéutico de la ciudad de Loja.

Los resultados se presentan a continuación, tomando en cuenta los objetivos de la investigación. Analizando de manera global la gestión administrativa y la calidad del servicio de atención al cliente, los resultados muestran, que los clientes que califican a dicho tipo de gestión como buena o muy buena, son también aquellos que le dan esos calificativos a la calidad del servicio, de acuerdo a la opinión del $59.2 \%$ y $100 \%$, respectivamente.

Al final se muestra que la gestión administrativa se relaciona de manera significativa (Sig. $<0.059$ ) con la calidad del servicio. La correlación positiva por su parte indica que en la medida que mejora la calificación de la gestión administrativa, mejorará la percepción por las funciones empresariales.

\section{Antecedentes de estudios}

En este rubro se reseñan los resultados y conclusiones de investigaciones que sirven de base para el desarrollo del presente estudio, partiendo desde los más antiguos hasta los más recientes.

Pérez (2002), en su publicación sobre la satisfacción al cliente como ventaja competitiva en farmacias de autoservicio de Venezuela, identificó y describió los factores de satisfacción del cliente. Para ello aplicó un cuestionario a los clientes que visitaron dicha empresa. Se utilizó la estadística descriptiva (porcentajes y gráficos de barras) para el análisis y presentación de los datos. Los resultados mostraron que los factores que determinan la satisfacción del cliente fueron: el sistema de distribución, calidad de los productos, precios y promoción. Adicionalmente se constató el desempeño de la gerencia de la empresa y el grado de competitividad de sus productos. 
Pérez (2008), comparó la satisfacción al cliente en farmacias de autoservicio de San Juan de los Morros, Estado Guárico. Las variables estudiadas fueron: atención al cliente, ventaja competitiva y satisfacción del cliente. La población y muestra está conformada por 22 empleados y 20 clientes de Farmacia Saas, 8 empleados y 20 clientes de Farmacia La Coromoto. Como técnicas de recolección de datos se utilizaron la revisión bibliográfica y la encuesta, esta última empleando como instrumentos dos cuestionarios: uno dirigido a los empleados de farmacias de 12 ítems y uno dirigido a los clientes de farmacias con 10 ítems; ambos instrumentos se diseñaron con preguntas cerradas de alternativas dicotómicas (Sí - No). La validez se determinó por un juicio de expertos y para la confiabilidad se aplicó una prueba piloto cuyos resultados fueron sometidos a los cálculos del coeficiente de Kuder y Richardson (KR-20), obteniendo 0,92 en el instrumento "A" y 0,81 en el instrumento "b"; es decir instrumentos altamente confiables. Se concluyó que el nivel de la satisfacción al cliente en Farmacia Saas en comparación con Farmacia La Coromoto, es más alto en términos de políticas de atención, filosofía de trabajo, conocimientos, habilidades y tecnología. Se recomendó realizar encuestas periódicas de mercadeo, así como mantener un estricto control sobre los procesos internos de atención al cliente.

Morales (2010), realizó un estudio para determinar la calidad de la atención que brinda la farmacia de emergencia del Hospital María Auxiliadora de Lima a los usuarios externos. Trabajó con una muestra de 378 usuarios de 44514 atenciones en el Departamento de Emergencia en el período Enero - Junio 2007. La muestra del personal de la farmacia fueron los trabajadores no químicos farmacéuticos. El 72,7 \% del personal comunicó que no son suficientes los recursos humanos para cumplir sus funciones y que no está garantizada la presencia del químico farmacéutico (QF), el 63,6\% no conoce el Manual de Organización y Funciones ni los Procedimientos de Atención, el 81,8\% no tiene reuniones frecuentes con la Química Farmacéutica responsable y el 63,6\% no acude a la Jefatura del Departamento de Farmacia cuando tiene algún problema, el 72,7\% no recibe capacitación en servicio desde el año 2001. De los usuarios el $41 \%$ alguna vez encontró, en la farmacia, todos los medicamentos que necesitaban, el 37\% indicó que el tiempo de espera de atención fue corto, el 50,3\% que el trato del personal, en la farmacia, fue bueno. Se concluye que en la farmacia de emergencia hay escasez de recursos humanos, escasez de infraestructura, falta de coordinación con la Química 
Farmacéutica y con la Jefatura, problemas de abastecimiento de medicamentos y material médico quirúrgico para atender a los usuarios.

Soto (2011), evaluó la gestión administrativa en centros médicos auspiciados por organismos internacionales, caso club rotario. La evaluación se centró en medir el cumplimiento de las fases del proceso administrativo, encontrándose que existe una marcada diferencia entre los establecimientos evaluados, ya que, a pesar de ser normados por la misma institución, aplican las fases administrativas de manera diferente y en algunos casos de manera incompleta, lo que no les permite lograr su principal objetivo de eficiencia. A partir de los hallazgos propone un modelo de gestión para mejorar la efectividad.

Taipe (2012), en el trabajo de investigación titulado "Sistema de Gestión Estratégica para las Cuentas por Cobrar en la Farmacia Cruz Azul Nº1, período del 1de enero al 30 de junio del 2009”, se planteó como objetivo evaluar la gestión administrativa e identificar la forma cómo se utiliza los recursos para la recaudación de la cartera vencida y disminuir el índice de morosidad. Aplicaron encuestas y entrevistas. En base a los resultados obtenidos se propuso la implementación de un Sistema de Gestión Estratégica.

\section{Marco teórico}

\section{Gestión}

Ivnacevich y Lorenzi (2006), sostienen que la gestión es el proceso emprendido por una o más personas para coordinar las actividades laborales de otras personas con la finalidad de lograr resultados de alta calidad que cualquier otra persona, trabajando sola, no podría alcanzar.

Laborda, Leopoldo; De Zuani, Elio (2009), argumenta que la gestión es la acción de gestionar y administrar una actividad profesional destinado a establecer los objetivos y medios para su realización, a precisar la organización de sistemas, con el fin de elaborar la estrategia del desarrollo y a ejecutar la gestión del personal. Asimismo, en la gestión es muy importante la acción, porque es la expresión de interés capaz de influir en una situación dada.

Campos, Segundo y Loza, Pedro (2011), retoma lo que cita el Diccionario Enciclopédico Gran Plaza y Jaime Ilustrado, señala que, "la gestión es la acción y efecto de gestionar o de administrar, es ganar, es hacer diligencias conducentes al logro de un negocio o de un deseo 
cualquiera". Desde otra perspectiva la define como "el conjunto de actividades de dirección y administración de una empresa". Además, manifiesta que gestión de empresas es un campo del conocimiento científico cuyo objeto de estudio son las empresas, explica su comportamiento científicamente y busca que se conduzcan con eficiencia por medio de técnicas y también del arte de quien las aplica.

Medina (2012), manifiesta que la palabra Gestión proviene del latín gestión, y hace referencia a la acción y al efecto de gestionar o de administrar. Gestionar es realizar diligencias conducentes al logro de un negocio o de un deseo cualquiera. Administrar, por otra parte, consiste en gobernar, dirigir, ordenar, disponer u organizar.

\section{Gestión administrativa}

Las teorías clásicas de la administración, como las de Taylor y Fayol, consideraban a las organizaciones como un sistema cerrado. Taylor consideraba que, para lograr eficiencia, las organizaciones deberían poner atención a los procesos operativos y Fayol al proceso administrativo. Los enfoques modernos consideran a las organizaciones como un sistema abierto. Desde esta perspectiva la empresa es un organismo social constituidas deliberadamente para lograr objetivos específicos (Hernández, Sergio; Palafox de Anda, Gustavo, 2012) y para ello cuentan con recursos (físicos, humanos y financieros); una estructura básica (marketing, operaciones, personal y finanzas) y son dirigidas y gestionados aplicando el proceso administrativo (planificación, organización, dirección y control) (Bernal, César A; Sierra, Hernán D., 2013) para cumplir con propósitos sociales externos en el entorno en que operan (Hernández, Sergio; Palafox de Anda, Gustavo, 2012). García del Junco, Julio; Casanueva Rocha, Cristóbal (2005) considera como un recurso más a los recursos inmateriales. Chiavenato (1995) al conceptualizar a la empresa como un sistema abierto considera al entorno como restricciones ambientales.

Se identifican como recursos básicos de la empresa: humanos, materiales y financieros. Los recursos humanos, están constituidos por las personas que aplican sus aptitudes y actitudes para la producción de bienes y/o servicios y se ubican en las unidades y niveles jerárquicos de la organización de acuerdo a las necesidades de la empresa y de las competencias que posee. Los recursos materiales, incluye a los insumos, materiales, maquinaria, equipo, herramientas, 
infraestructura, necesarios para la producción de bienes y/o servicios. Los recursos financieros, se refiere a los recursos económicos propios que permiten comprar los recursos y gestionar la estructura básica. García del Junco, Julio; Casanueva Rocha, Cristóbal (2005), considera que otro recurso son los recursos inmateriales o también denominados "activos intangibles" y entre ellos están la imagen de la empresa, su cultura, la marca, las patentes, sus concesiones, etc.

Toda organización tiene una estructura básica que gestionar y comprende: gestión del marketing, gestión de operaciones, gestión de finanzas y gestión del recurso humano. En el enfoque de sistemas la gestión del marketing o gestión comercial es el encargado de recibir los productos del subsistema producción y realizar los procesos de intercambios correspondientes (García del Junco, Julio; Casanueva Rocha, Cristóbal, 2005). La gestión del marketing, se concentra en la búsqueda de información sobre las necesidades del mercado potencial con propósito de satisfacerlas. El marketing operativo se concentra en ejecutar un plan de acción para satisfacer las necesidades de un segmento de mercado seleccionado. El plan de acción contiene decisiones sobre cuatro variables conocidas como el marketing mix: producto, precio, plaza y promoción.

La gestión de operaciones, inicialmente denominado gestión de la producción. Esta última nominación fue remplazada por la primera, por ser un concepto restrictivo, que incluía sólo a organizaciones que transforman los recursos en productos y excluía a las empresas prestadoras de servicio. En el enfoque sistémico es el conjunto de actividades orientadas a transformar un conjunto de factores o recursos (inputs) en productos o servicios (outputs). La función de producción o de operaciones pone énfasis en tres aspectos fundamentales: la elección y control de las entradas; el diseño y control del proceso productivo; y el control de las salidas (calidad, utilidad, rendimiento y productividad) (García del Junco, Julio; Casanueva Rocha, Cristóbal, 2005).

La gestión de finanzas, es el conjunto de actividades orientadas a programar, controlar u regular los flujos de entradas y salidas de dinero de la empresa. La gestión de las finanzas comprende la función de inversión y la función de financiamiento. La primera se refiere a los problemas y decisiones sobre el empleo del dinero; y el segundo sobre problemas y decisiones sobre cómo obtener los recursos que la empresa necesita (García del Junco, Julio; Casanueva Rocha, Cristóbal, 2005). 
La gestión de finanzas, es el conjunto de actividades orientadas a programar, controlar u regular los flujos de entradas y salidas de dinero de la empresa. La gestión de las finanzas comprende la función de inversión y la función de financiamiento. La primera se refiere a los problemas y decisiones sobre el empleo del dinero; y el segundo sobre problemas y decisiones sobre cómo obtener los recursos que la empresa necesita (García del Junco, Julio; Casanueva Rocha, Cristóbal, 2005).

La gestión de recursos humanos, comprende los subsistemas de alimentación (planificación, reclutamiento y selección de los recursos humanos); de aplicación (descripción y análisis de cargos y evaluación del desempeño humano) de mantenimiento (compensación, beneficios sociales, higiene y seguridad industrial y relaciones laborales); de desarrollo (capacitación y desarrollo de personal y desarrollo organizacional); de control (sistemas de información y auditorías de recursos humanos) (Chiavenato, 1995).

\section{Calidad}

Ivnacevich y Lorenzi (2006), manifiestan que la calidad es la totalidad de los rasgos y las características de un producto o servicio que se refieren a su capacidad para satisfacer necesidades expresadas o implícitas.

Fragas (2012), en su trabajo de investigación sobre la propuesta de procedimiento de costos de la calidad de Audita S.A. Sucursal Cienfuegos, retoma las conceptualizaciones de calidad de algunos autores como el de Kaoru Ishikawa (1988), quien define a la calidad como "desarrollar, diseñar, manufacturar y mantener un producto de calidad que sea el más económico, el útil y siempre satisfactorio para el consumidor". E.W. Deming (1989), establece que es el "grado predecible de uniformidad y fiabilidad a un bajo costo y que se ajuste a las necesidades del mercado. La calidad no es otra cosa más que "una serie de cuestionamiento hacia una mejora continua". Philip Crosby (1989), puntualiza que la calidad es entregar a los clientes y a los compañeros de trabajos, productos y servicios sin defectos y hacerlo a tiempo.

Yamaguchi (1989), citado por Armas (2006), precisa que la calidad es el conjunto de propiedades o características que definen su actitud para satisfacer necesidades establecidas. J. M. Juran (1993a), define que, Calidad es el conjunto de características que satisfacen las necesidades de 
los clientes, además calidad consiste en no tener deficiencias. La calidad es la adecuación para el uso satisfaciendo las necesidades del cliente.

Feigenbaum (1971-1994), considera que calidad es un sistema eficaz para integrar los esfuerzos de mejora de la gestión, de los distintos grupos de la organización para proporcionar productos y servicios a niveles que permitan la satisfacción del cliente, a un costo que sea económico para la empresa, agregando posteriormente: calidad es la resultante de una combinación de características de ingeniería y de fabricación, determinantes del grado de satisfacción que el producto proporcione al consumidor durante su uso.

Hansen (1996) citado por López (2005), señala que "la calidad es el grado o nivel de excelencia, es una medida de lo bueno de un producto o servicio". Cuatrecasas (1999), considera la calidad como: el conjunto de características que posee un producto o servicio obtenidos en un sistema productivo, así como su capacidad de satisfacción de los requisitos del usuario. La norma (ISO 9000:2005), plantea que es el grado en el que un conjunto de características inherentes cumple con los requisitos.

\section{Calidad del servicio}

De acuerdo a lo que establece (Berzosa, Beatriz; Cámara, Luis \& otros, 2005) en su publicación sobre la Fundación de Asistencia Técnica para el Desarrollo español, indica, que el ámbito de la gestión de las organizaciones, los primeros planteamientos de la calidad surgen con los principios científicos de la organización y división del trabajo de comienzos del siglo XX (taylorismo, fordismo), y su aplicación a los procesos de producción en cadena para aumentar la producción y abaratar costes, tratando de mejorar la eficiencia empresarial. Este modelo se caracterizaba principalmente por la especialización de cada operario, una clara separación de las funciones de planificación, ejecución y control y la existencia de detalladas normas y regulaciones sobre cómo debe operar el sistema o la organización.

\section{Metodología}

El diseño de la presente investigación es no experimental, el tipo de investigación, el presente estudio reúne las condiciones metodológicas de una investigación aplicada, en razón que se aplicó el enfoque Empírico - Inductivo - Subjetivo de la investigación, siguiendo la clasificación 
de José Padrón. Se aplicó la teoría del proceso administrativo y la teoría de la calidad del servicio, a fin elaborar conocimiento sobre las variables propuestas.

De acuerdo a la naturaleza del estudio de la investigación, reúne nivel de las características de un estudio correlacional, siguiendo el criterio de clasificación de Hernández (2009). Los estudios correlacionales, buscan establecer relaciones entre las variables, sin identificar dependencia entre ellas.

La población de farmacias es de 204, de los cuales 68 son directivos de la empresa y 136 son personal del servicio de atención al cliente. Por ser una población manejable se censo a todos ellos

Las principales técnicas de investigación que se utilizaron para la recolección de la información fueron: cuestionario y análisis bibliográfico. El análisis bibliográfico se utilizó para la elaboración del marco de referencia, utilizando una guía de análisis bibliográfico.

Se aplicó dos cuestionarios, con el mismo formato, a los directivos y al personal del servicio de atención al cliente, estructurados en escala de Likert, los que fueron validados según el criterio de “juicio de expertos", que arrojaron un índice de validez de 2.71, que califica como una validez fuerte. De acuerdo a las sugerencias de los evaluadores se eliminó la pregunta 1 y se replantearon las preguntas 22, 26, 36 y 91.

Para la confiabilidad, se utilizó el índice de consistencia y coherencia interna, Alfa de Cronbach; los valores de éste índice para el cuestionario de gestión de recursos y capacidades en el mercado farmacéutico, son respectivamente de 0.974 y 0.969, que caen en el rango de confiabilidad muy alta, lo que garantiza que la aplicación del instrumento proporcionará información confiable para cumplir con los objetivos planteados en la investigación.

Para calificar la escala se utilizaron cinco alternativas de respuesta, a las cuales se les asignó valores del 0 al 4; como el promedio de esta escala es de dos puntos, entonces valores de 3 o 4 puntos evidencian una valoración favorable, mientras que valores iguales o superiores a los dos puntos evidencian una valoración negativa. 
Para el análisis de los datos se utilizó estadística descriptiva como promedios y porcentajes para el análisis individual de las variables y para establecer la relación entre las variables se calculó el índice de correlación de Spearman por tratarse de variables de naturaleza cualitativa.

\section{Resultados}

Los resultados se presentan a continuación, dando en primer lugar una visión general de la percepción que tienen los clientes por cada una de las variables, para luego describir los resultados asociados a los objetivos de la i investigación.

\section{Gestión de recursos y capacidades en el mercado farmacéutico}

En la tabla 1 se presenta la información relacionada a los indicadores de gestión de los recursos y capacidades en el mercado farmacéutico, según la percepción de los clientes.

Esta tabla muestra la percepción que tienen los clientes por la gestión administrativa, la que incluye la gestión de recursos y de las capacidades. Los promedios cercanos a 3 puntos dejan en evidencia que los clientes califican de manera favorable, la gestión de recursos y capacidades directivas y de marketing, así como las del potencial humano y de operaciones; por el contrario, la gestión de las capacidades financieras no es bien valorada, según el promedio $(2,4)$ que se acerca a los 2 puntos, convirtiéndose en uno de los aspectos menos valorados de la gestión. Analizando de manera conjunta estos resultados en la dimensión, gestión de recursos y capacidades, ésta logra una valoración favorable, de acuerdo al promedio $(2,6)$ cercano a los 3 puntos.

Evaluando de forma holística la gestión, el estudio da cuenta que ésta es favorable, según lo indica el promedio $(2,6)$ cercano a los 3 puntos.

Tabla 1. Percepción de los clientes por los aspectos de la gestión de recursos y capacidades en el mercado farmacéutico de la ciudad de Loja 


\begin{tabular}{|l|c|c|}
\hline \multicolumn{1}{|c|}{ Indicadores } & media & desv. Tipica \\
\hline Recursos y Capacidades & $\mathbf{2 , 6}$ & $\mathbf{0 , 6}$ \\
\hline Recursos y capacidades directivas & 2,7 & 0,5 \\
Recursos y capacidades de marketing & 2,7 & 0,6 \\
Recursos y capacidades financieras & 2,4 & 0,6 \\
Recursos y capacidades del potencial humano & 2,5 & 0,6 \\
Recursos y capacidades de operaciones & 2,5 & 0,8 \\
\hline
\end{tabular}

Fuente: Cuestionario aplicado a los clientes

Elaboración: Los Autores

\section{Contrastación de la hipótesis}

La gestión de los recursos y capacidades se relaciona significativamente con la calidad del servicio de atención al cliente en el mercado farmacéutico de la ciudad de Loja, año 2016, desde la perspectiva de los trabajadores.

En la tabla 2 el análisis de correlación deja en evidencia que la gestión de los recursos y capacidades directivas, capacidades de marketing, capacidades financieras, capacidades del potencial humano, capacidades de operaciones y en general la Gestión de Recursos y capacidades se relacionan de manera significativa $($ Sig. $<0.05)$ con la calidad del servicio que brinda el mercado farmacéutico de la ciudad de Loja, lo que conduce a aceptar la hipótesis de investigación.

Tabla 2. Correlación entre las dimensiones de la gestión y la calidad

\begin{tabular}{|c|c|c|}
\hline Gestión & Spearman & Calidad \\
\hline \multirow[t]{2}{*}{ Recursos y capacidades directivas } & $r$ & $0,454(* *)$ \\
\hline & sig & 0,000 \\
\hline \multirow[t]{2}{*}{ Recursos y capacidades de marketing } & $\mathrm{r}$ & $0,599(* *)$ \\
\hline & sig & 0,000 \\
\hline \multirow[t]{2}{*}{ Recursos y capacidades financieras } & $r$ & $0,559(* *)$ \\
\hline & sig & 0,000 \\
\hline \multirow[t]{2}{*}{ Recursos y capacidades del potencial humano } & $r$ & $0,543(* *)$ \\
\hline & sig & 0,000 \\
\hline \multirow[t]{2}{*}{ Recursos y capacidades de operaciones } & $r$ & $0,620(* *)$ \\
\hline & sig & 0,000 \\
\hline \multirow[t]{2}{*}{ Recursos y capacidades } & $r$ & $0,674(* *)$ \\
\hline & sig & 0,000 \\
\hline
\end{tabular}

Fuente: Cuestionario aplicado a los clientes

Elaboración: Los Autores 
** La correlación es significativa al nivel 0,01 (bilateral).

\section{Relación entre la gestión administrativa con la calidad de los servicios de atención al cliente en el mercado farmacéutico}

La tabla 3 muestra, la relación que existe entre la gestión administrativa con la calidad del servicio de atención al cliente en el mercado farmacéutico de la ciudad de Loja.

Al analizar de manera global la gestión administrativa y la calidad del servicio, los resultados muestran que los clientes que califican a dicha gestión como deficiente o regular, son mayormente los que también califican a la calidad del servicio en dichos niveles, de acuerdo a la opinión del $57.1 \%$ y $51.6 \%$, respectivamente; por el contrario, los clientes que califican a dicho tipo de gestión como buena o muy buena, son también aquellos que le dan esos calificativos a la calidad del servicio, de acuerdo a la opinión del 59.2\% y 100\%, respectivamente.

Tabla 3. Relación entre la Gestión Administrativa y la calidad del servicio de atención al cliente

\begin{tabular}{|l|c|c|c|c|c|c|c|c|c|c|}
\hline Gestión & \multicolumn{10}{|c|}{ Calidad } \\
\hline de Recursos y & \multicolumn{1}{|c|}{ Deficiente } & \multicolumn{1}{|c|}{ Regular } & \multicolumn{1}{c|}{ Buiena } & \multicolumn{2}{|c|}{ Muy Buena } & \multicolumn{2}{c|}{ Total } \\
\cline { 2 - 13 } Capacidades & Nro & $\%$ & Nro & $\%$ & Nro & $\%$ & Nro & $\%$ & Nro & $\%$ \\
\hline Deficiente & 4 & $57,1 \%$ & 3 & $42,9 \%$ & 0 & $0,0 \%$ & 0 & $0,0 \%$ & 7 & $100 \%$ \\
Regular & 12 & $13,2 \%$ & 47 & $51,6 \%$ & 32 & $35,2 \%$ & 0 & $0,0 \%$ & 91 & $100 \%$ \\
Buena & 0 & $0,0 \%$ & 20 & $19,4 \%$ & 61 & $59,2 \%$ & 22 & $21,4 \%$ & 103 & $100 \%$ \\
Muy buena & 0 & $0,0 \%$ & 0 & $0,0 \%$ & 0 & $0,0 \%$ & 3 & $100,0 \%$ & 3 & $100 \%$ \\
Total & 16 & $7,8 \%$ & 70 & $34,3 \%$ & 93 & $45,6 \%$ & 25 & $12,3 \%$ & 204 & $100 \%$ \\
\hline
\end{tabular}

Fuente: Cuestionario aplicado a los clientes

Elaboración: Los Autores

\section{Discusión}

El presente estudio compara los hallazgos de Pérez 2002, en su publicación sobre la satisfacción al cliente como ventaja competitiva en farmacias de autoservicio de Venezuela, identificó y describió los factores de satisfacción del cliente. Los resultados mostraron que los factores que determinan la satisfacción del cliente fueron: el sistema de distribución, calidad de los productos, precios y promoción. Adicionalmente se constató el desempeño de la gerencia de la empresa y el grado de competitividad de sus productos. Mientras que en el trabajo objeto de estudio en la parte 
de percepción de los clientes por los aspectos de calidad del servicio de atención al cliente en el mercado farmacéutico de la ciudad de Loja, los clientes tienen una percepción favorable de los elementos tangible de la fiabilidad, capacidad de respuesta y empatía del servicio. Se puede dejar ver que la investigación actual contrasta con la realizada anteriormente, por cuanto la presente mantiene un resultado de satisfacción de la atención al cliente que proviene de la capacidad que tiene en sus recursos humanos que atienden al cliente, mientras que la investigación anterior califica poniendo énfasis al producto, sus procesos establecidos, precios y promociones; desconociendo la fortaleza del recurso humano.

Morales (2010), realizó un estudio para determinar la calidad de la atención que brinda la farmacia de emergencia del Hospital María Auxiliadora de Lima a los usuarios externos. Se concluye que en la farmacia de emergencia hay escasez de recursos humanos, escasez de infraestructura, falta de coordinación con la Química Farmacéutica y con la Jefatura, problemas de abastecimiento de medicamentos y material médico quirúrgico para atender a los usuarios. En la investigación actual se observa en el análisis de la relación entre la gestión de recursos y capacidades de operación y la calidad del servicio, que en la medida que se valora más a la gestión de los recursos y capacidades de operación, mejora la calificación de la calidad de los servicios. Al hacer la relación se puede decir que existe de alguna manera cierta semejanza en los resultados, debido a que en la investigación de Morales señala que la escasez de los recursos, no le permite entregar el servicio de calidad, es decir que se entiende que están inmersos la relación entre la gestión de los recursos y capacidades que se mencionan en la investigación actual.

Castellanos, Susie y González, Pamela (2010), compara la calidad de los servicios en farmacias con enfoque tradicional y las de autoservicio en el municipio de Maracaibo. Realizaron revisión documental de fuentes secundarias y aplicaron una lista de verificación a las farmacias con escala dicotómica para identificar sus características y una entrevista estructurada con escala de Likert a los usuarios de las farmacias. Los resultados arrojaron la existencia de una mediana calidad de servicio de las farmacias tradicionales y una alta calidad para las farmacias de autoservicio en el municipio Maracaibo del estado Zulia, por lo que se recomienda a las tradicionales enfocarse en sus puntos débiles para superarlos tomando en cuenta las fortalezas de las farmacias de autoservicio. El aporte que entrega este artículo a la presente investigación es demasiado general, por cuanto su resultado manifiesta que la farmacia tradicional debe tomar como ejemplo a la 
farmacia de autoservicio para corregir sus debilidades, poro no le hace conocer las causas de su fracaso ni y las alternativas de solución.

Soto (2011), evaluó la gestión administrativa en centros médicos auspiciados por organismos internacionales, caso club rotario. La evaluación se centró en medir el cumplimiento de las fases del proceso administrativo, encontrándose que existe una marcada diferencia entre los establecimientos evaluados, ya que, a pesar de ser normados por la misma institución, aplican las fases administrativas de manera diferente y en algunos casos de manera incompleta, lo que no les permite lograr su principal objetivo de eficiencia. A partir de los hallazgos propone un modelo de gestión para mejorar la efectividad. Para analizar el aporte de Soto, en su investigación se toma de la investigación desarrollada actualmente la relación entre gestión administrativa y la calidad del servicio que de algún modo se refiere al manejo del proceso administrativo en las farmacias. Esta relación consiste en que la calidad del servicio puede estar enfocada desde el punto de vista de la gestión administrativa que no es otra cosa que al manejo y aplicación del proceso administrativo. Partiendo de ese criterio se considera que los clientes estarían calificando la labor de gestión que aprovechando la propuesta de una de las investigaciones tomarían el modelo de gestión para mejorar la efectividad.

Taipe (2012), en el trabajo de investigación titulado "Sistema de Gestión Estratégica para las Cuentas por Cobrar en la Farmacia Cruz Azul Nº1, período del 1de enero al 30 de junio del 2009”, se planteó como objetivo evaluar la gestión administrativa e identificar la forma cómo se utiliza los recursos para la recaudación de la cartera vencida y disminuir el índice de morosidad. Aplicaron encuestas y entrevistas. En base a los resultados obtenidos se propuso la implementación de un Sistema de Gestión Estratégica. El trabajo de Taipe se lo tomaría como un complemento de la anterior, es decir evaluar la gestión administrativa para determinar las debilidades y corregirlas con la aplicación del sistema de gestión estratégica.

González y Bermúdez (2014), estudió la calidad de los servicios farmacéuticos comunitarios del área de salud Julián Grimau, en el municipio Santiago de Cuba, en el período comprendido entre enero y febrero del 2010, tomando como referencias las normativas oficiales del país. Para evaluar la calidad se establecieron indicadores y estándares para la estructura, proceso y resultados, así como se calculó el coeficiente de calidad. Los resultados mostraron que los 
servicios farmacéuticos evaluados no poseen calidad porque presentaron insuficiente disponibilidad de profesionales farmacéuticos calificados, factores que contribuyeron a la insatisfacción de los pacientes, facultativos y profesionales farmacéuticos del área de salud objeto de análisis. Se pudo comparar la investigación de González y Bermúdez con la relación entre la gestión de los recursos y capacidades del potencial humano y la calidad del servicio de atención al cliente analizado en la presente investigación en la que se determinó que existe relación directa por que sus resultados arrojan que la calidad del servicio se valora tomando en consideración el potencial profesional de las servidores de las farmacias, en el trabajo realizado anteriormente sobre la calidad de los servicios farmacéuticos comunitarios del área de salud Julián Grimau, se determina la insuficiente disponibilidad de profesionales farmacéuticos los conduce a la no existencia de calidad en el servicio, mientras que en el actual trabajo investigativo se determina califican como deficiente o muy deficiente a la gestión de los recursos y capacidades del potencial humano, como se observa en el $100 \%$ y $60 \%$ respectivamente.

\section{Conclusiones}

El análisis de la información permite llegar a las siguientes conclusiones:

Analizando las variables de la Gestión de las capacidades directivas y los recursos y Calidad de los servicios de atención al cliente, se pudo llegar a las siguientes conclusiones:

El análisis de correlación deja en evidencia que la gestión de los recursos y capacidades directivas, capacidades de marketing, capacidades financieras, capacidades del potencial humano, capacidades de operaciones y en general la gestión de recursos y capacidades se relacionan de manera significativa (Sig.<0.05) con la calidad del servicio que brinda el mercado farmacéutico de la ciudad de Loja, lo que conduce a aceptar la hipótesis de investigación., La gestión de los recursos y capacidades se relaciona significativamente con la calidad del servicio de atención al cliente en el mercado farmacéutico de la ciudad de Loja - Ecuador, año 2016, desde la perspectiva de los trabajadores.

Los resultados de la prueba indican que las condiciones generales del entorno se relacionan de manera significativa (Sig. $<0.05$ ) con la calidad del servicio que brinda el mercado farmacéutico de la ciudad de Loja; este resultado permite validar la hipótesis de investigación, La gestión de las condiciones generales del entorno se relaciona significativamente con la calidad del servicio 
de atención al cliente en el mercado farmacéutico de la ciudad de Loja - Ecuador, año 2016. Desde la perspectiva de los trabajadores.

El análisis de correlación, dejan en evidencia que las dimensiones de las condiciones específicas del sector, competencia, proveedores, clientes y condiciones específicas del sector, se relacionan de manera significativa (Sig. $<0.05$ ) con la calidad del servicio; este resultado conduce a aceptar la hipótesis de investigación, La gestión de las condiciones específicas del entorno se relaciona significativamente con la calidad del servicio de atención al cliente en el mercado farmacéutico de la ciudad de Loja - Ecuador, año 2016, desde la perspectiva de los trabajadores.

\section{Referencias bibliográficas}

Bernal, César A; Sierra, Hernán D. (2013). Proceso administrativo para las organizaciones del siglo XXI. Colombia: PEARSON.

Berzosa, Beatriz; Cámara, Luis \& otros. (2005). Calidad del Servicio. Obtenido de La Gestión de la Calidad; Guía para la adaptación del modelo EFQM de excelencia a entidades no lucrativas que prestan servicios de inserción socio laboral.: http://www.kalidadea.org/castellano/materiales/gestion\%20estrategica\%20y\%20calidad/gestion_ calidad\%20CIDEAL.pdf

Campos, Segundo; Loza, Pedro. (2011). GESTION. Incidencia de la Gestión Administrativa de la Biblioteca Municipal "Pedro Moncayo" de la ciudad de Ibarra en Mejora de la Calidad de Servicios y Atención a los usuarios en el año 2011. Propuesta Alternativa. Ibarra.

Castellanos, Susie \& González, Pamela. (2010). Calidad de Servicio en farmacias tradicionales y de autoservicio: Estudio de Caso. Scielo, 52.

Chiavenato, I. (1995). Administración de recursos humanos. Colombia: Mc Graw Hill.

Fragas, L. (2012). La calidad. Obtenido de http://www.eumed.net/librosgratis/2013/1283/1283.pdf

García del Junco, Julio; Casanueva Rocha, Cristóbal. (2005). Fundamentos de gestión empresarial. Madrid. España: Pirámide. 
González, A., \& Bermúdez, I. y. (2014). Calidad de los servicios farmacéuticos comunitarios del área de la salud Julián Grimau. MEDICOS, 6.

Hernández, Sergio; Palafox de Anda, Gustavo. (2012). Administración: teoría, proceso, áreas funcionales y estrategias para la competitividad. México: Mc Graw Hill.

Ivnacevich, J., \& Lorenzi, P. \&. (2006). GESTION: CALIDAD Y COMPETITIVIDAD. Colombia: Irwin. Pàg. 12. Tomo I.

Laborda, Leopoldo; De Zuani, Elio. (2009). FUNDAMENTOS DE GESTIÓN EMPRESARIAL. Argentina: Valleta Ediciones. Pág. 28.

Medina, J. (Abril de 2012). GESTION. Modelo de Gestión Administrativa para el Gobierno de la Parroquia de Yaruquì, Cantón Quito, Provincia de Pichincha. Quito.

Morales, M. d. (2010). Calidad de atención en la farmacia de emergencia del Hospital María Auxiliadora de Lima. Obtenido de Calidad de atención en la farmacia de emergencia del Hospital María Auxiliadora de Lima.: http://sisbib.unmsm.edu.pe/bvrevistas/rev_academia/2011_n2/pdf/a15v18n2.pdf. Pàg. 41

Pérez, A. D. (2008). Satisfacción de las necesidades del cliente. Obtenido de Satisfacción al cliente como ventaja competitiva en farmacias de autoservicio (Venezuela). Pág. 2: http://www.monografias.com/trabajos66/satisfaccion-cliente-farmacias-autoservicio/satisfaccioncliente-farmacias-autoservicio2.shtml

SOTO, J. (Septiembre de 2011). Evaluación de la Gestión Administrativa en Centros Médicos auspiciados por Organismos Internacionales, caso Club Rotario. Quito, Ecuador. Pàg. 3 y 16.

Taipe, L. (Enero de 2012). Sistemas de Gestión Estratégica para las cuentas por cobrar en la Farmacia Cruz Azul No. 1, ubicada en la ciudad de Latacunga al período del $1^{\text {a }}$ de enero al 30 de junio de 2009. Latacunga, Ecuador. Pág. 5. 